\title{
Psychosocial Comorbidities and Health Status Among Adults with Moderate-to-Severe Atopic Dermatitis: A 2017 US National Health and Wellness Survey Analysis
}

\author{
Shawn G. Kwatra · David Gruben · Selwyn Fung $\cdot$ Marco DiBonaventura (1)
}

Received: November 16, 2020 / Accepted: January 15, 2021 / Published online: February 8, 2021

(c) The Author(s) 2021

\section{ABSTRACT}

Introduction: Atopic dermatitis (AD) is associated with sleep difficulties, depression, and anxiety. We evaluated the relationship between these psychosocial comorbidities and health outcomes among adults with moderate-to-severe $\mathrm{AD}$ in the USA.

Methods: Data were analyzed from the 2017 US National Health and Wellness Survey. Respondents with a physician diagnosis of $\mathrm{AD}$ or eczema with moderate-to-severe AD based on a Dermatology Life Quality Index score of 6 or more were included. Generalized linear models were used to examine the relationship between psychosocial comorbidities (sleep difficulties and anxiety based on self-report, depression based on the Patient Health Questionnaire-9) and health outcomes [the 36-item Short Form Health Survey, version 2; EuroQol five-

Supplementary Information The online version contains supplementary material available at https:// doi.org/10.1007/s12325-021-01630-z.

S. G. Kwatra

Department of Dermatology, Johns Hopkins School of Medicine, Baltimore, MD, USA

D. Gruben

Pfizer Inc., Groton, CT, USA

S. Fung $\cdot$ M. DiBonaventura $(\bowtie)$

Pfizer Inc., New York, NY, USA

e-mail: marco.dibonaventura@pfizer.com dimension, five-level; Work Productivity and Activity Impairment questionnaire; and healthcare resource utilization (HRU)].

Results: Among respondents with moderate-tosevere $\mathrm{AD}(N=1017), 56.6 \%, 70.7 \%$, and $60.9 \%$ reported sleep difficulties, depression, and anxiety, respectively. These comorbidities were significantly associated with reduced physical and mental component summary scores and increased overall work impairment $(P<0.05$ for all). Increased HRU was also observed.

Conclusion: Psychosocial comorbidities were frequently reported by respondents with moderate-to-severe $\mathrm{AD}$ and were significantly associated with health status, work loss, and HRU.

Keywords: Anxiety; Atopic dermatitis; Depression; Healthcare resource utilization; Health-related quality of life; Itch; Pruritus; Sleep difficulties; Work impairment 


\section{Key Summary Points}

Why carry out this study?

Psychosocial comorbidities, including sleep difficulties, depression, and anxiety, are common among patients with moderate-to-severe atopic dermatitis (AD) in the USA.

This analysis was conducted to evaluate the relationship between these psychosocial comorbidities and health outcomes among adults with moderateto-severe $\mathrm{AD}$ in the USA.

\section{What was learned from this study?}

These AD-related comorbidities-sleep difficulties, depression, and anxiety-are associated with a reduction in health status, health-related quality of life, and productivity in daily activities.

These comorbidities can also result in substantial economic burden based on direct healthcare costs and indirect costs from lost productivity.

\section{DIGITAL FEATURES}

This article is published with digital features, including a summary slide, to facilitate understanding of the article. To view digital features for this article go to https://doi.org/10.6084/ m9.figshare.13574552.

\section{INTRODUCTION}

Atopic dermatitis (AD) is a chronic, inflammatory disease of the skin characterized by intense pruritus and eczematous lesions [1]. AD affects $20 \%$ of children and $10 \%$ of adults in the USA $[2,3]$ and is significantly associated with decreased levels of health-related quality of life (HRQoL) [4]. Debilitating pruritus, the central symptom of $\mathrm{AD}$, can be particularly disruptive to sleep [5]. Coupled with the social stigma of visible skin lesions, AD is associated with higher rates of depression and anxiety among patients $[6,7]$.

The substantial economic burden of $\mathrm{AD}$ is based on direct healthcare costs and indirect costs from lost productivity. Recent US claims analyses have shown that total annual per patient costs were approximately $\$ 15,000-20,000$ for those with moderate-tosevere disease, with the largest share of costs attributed to outpatient visits [8-10]. AD is also significantly associated with work loss and sick leave, and preliminary evidence suggests that AD can affect occupational choices [11]. In the USA, employed adults with AD experience approximately three times the level of absenteeism compared with matched controls and report that more than $25 \%$ of their work time is missed or rendered ineffective because of their health [4].

The aim of this study was to quantify the extent to which the psychosocial comorbidities of sleep difficulties, depression, and anxiety can further contribute to the humanistic and economic burden of disease experienced by patients with $\mathrm{AD}$. The current study shows the prevalence of each of these psychosocial comorbidities among patients with moderateto-severe $\mathrm{AD}$ in the USA and examines how the presence and severity of these comorbidities relate to health outcomes, including health status, HRQoL, work and activity impairment, and healthcare resource utilization (HRU). These data can inform overall management of $\mathrm{AD}$ to alleviate the burden it places on patients, caregivers, employers, and the healthcare system.

\section{METHODS}

\section{Data Source}

This study involves an analysis of the crosssectional 2017 National Health and Wellness Survey (NHWS), an annual, cross-sectional, Internet-based survey of adults (at least 18 years old) conducted across several countries. The current study used the US data set $(N=75,004)$. 
Potential respondents to the NHWS were recruited through various online panels (e.g., Lightspeed Research) and were sampled in a random stratified manner to mimic the demographic distributions of the adult US population based on the current population survey from the US Census [12].

Potential respondents received an email invitation to complete the survey. Respondents who provided consent through a signed and dated electronic document completed a series of screening questions to assess eligibility. Eligible respondents aged at least 18 years old completed the survey, which took approximately 30-45 min. Respondents who completed the survey were compensated with points that were valued between $\$ 5$ and $\$ 10$.

\section{Sample}

Respondents who completed the NHWS were included in the analyses if they self-reported a physician diagnosis of "atopic dermatitis" or "eczema" and scored 6 or more on the Dermatology Life Quality Index (DLQI), which suggested a "moderate" to "extremely large" effect on a patient's quality of life [13].

\section{Measures}

\section{Demographics and Health History}

Respondents answered demographic questions regarding age, sex, race/ethnicity, marital status, income, and employment status; they also reported general health history information, including smoking status, alcohol consumption, and the number of years since being diagnosed with AD. Respondents self-reported their height and weight to calculate a body mass index category and their comorbidities to calculate a Charlson comorbidity index [14].

\section{Psychosocial Comorbidities}

Respondents self-reported whether they experienced either "insomnia" or "sleep difficulties" in the past 12 months, and for each reported the level of severity ("mild," "moderate," or "severe"). The severity of sleep difficulties was defined using the highest self-reported severity rating of either insomnia or sleep difficulties in the past 12 months (mild, moderate, or severe). For respondents who did not report either condition, severity was set as "no sleep difficulties," generating four levels of severity for this categorical variable.

Respondents also completed the Patient Health Questionnaire-9 (PHQ-9) [15], a validated nine-item instrument used to assess the severity of depression in the last 2 weeks: 0-4, "none/minimal depression"; 5-9, "mild depression"; 10-14, "moderate depression"; 15-19, "moderately severe depression"; and 20-27, "severe depression." Respondents also selfreported whether they had experienced anxiety in the past 12 months.

\section{Health Outcomes}

The 36-item Short Form Health Survey, version 2 (SF-36v2) [16]; EuroQol five-dimension, five-level questionnaire (EQ-5D-5L) [17]; and the Work Productivity and Activity Impairment questionnaire-General Health version (WPAIGH) [18] were all administered as part of the NHWS. The SF-36v2 is a generic health status instrument that has two summary scores (physical component summary and mental component summary) and eight domain scores (bodily pain, vitality, general health, physical functioning, physical role functioning, emotional role functioning, mental health, and social functioning). All summary and domain scores are normalized using the QualityMetric system, with the population mean and standard deviation set to 50 and 10, respectively; higher scores indicate better health status [16]. The EQ$5 \mathrm{D}-5 \mathrm{~L}$ is a generic HRQoL measure that produces a health utility index score from 0 to 1 , with higher scores indicating better quality of life (QoL) [17]. The WPAI-GH is a six-item validated tool to assess the impact of health on one's work and leisure activities. It includes four metrics: absenteeism (percentage of work time missed because of one's health in the past 7 days), presenteeism (percentage impairment experienced while at work in the past 7 days because of one's health), overall work 
impairment (combination of absenteeism and presenteeism), and activity impairment (percentage impairment in daily activities because of one's health in the past 7 days) [18]. Respondents also self-reported HRU [i.e., number of healthcare provider visits, emergency room (ER) visits, and hospitalizations] in the past 6 months.

\section{Statistical Analyses}

The prevalence of psychosocial comorbidities among respondents with $\mathrm{AD}$ was reported descriptively. Generalized linear models were used to examine the association between psychosocial comorbidity categories and each health outcomes variable. For normally distributed outcomes (SF-36v2 component summary and domain scores and EQ-5D-5L index scores), a normal distribution with an identity link function was used. For positively skewed variables (WPAI-GH scores and HRU), negative binomial distributions with a log-link function were used. Age and sex were selected covariates a priori. Any other demographic or health characteristics which differed across analysis groups at $P<0.1$ were included as covariates. The presence of comorbidities (including diabetes) was included as a covariate as part of the Charlson comorbidity index, and other atopic conditions (which are not represented in the Charlson comorbidity index) were included separately. All models controlled for age, sex, race/ethnicity, education, income, employment status, body mass index, smoking status, alcohol use, Charlson comorbidity index score, and presence of other atopic conditions. Adjusted means were reported on the original metric of each variable for each level of the comorbidity category. The reference category was set to be the lowest level of each psychosocial comorbidity. Statistical significance was based on $P<0.05$ relative to this reference category.

\section{Compliance with Ethics Guidelines}

The institutional review board at each study site approved the study protocol, and written informed consent was provided by parents/legal guardians. The study was conducted in accordance with the protocol, local legal and regulatory requirements, and the general principles set forth in the International Ethical Guidelines for Biomedical Research Involving Human Subjects, International Conference on Harmonisation Guideline for Good Clinical Practice, and the Declaration of Helsinki. The protocol and study materials associated with the original fielding of the 2017 NHWS were reviewed by the Pearl Institutional Review Board (Indianapolis, IN) and granted exemption status.

\section{RESULTS}

\section{Sample Characteristics and Prevalence of Psychosocial Comorbidities}

A total of 1017 respondents met criteria for having moderate-to-severe $\mathrm{AD}$ and were included in the analyses. Respondents were predominantly female $(73.6 \%)$ with a mean age of 37.4 years (SD 14.5 years) and were diverse with respect to race and ethnicity $(41.9 \%$ nonHispanic white, $17.3 \%$ non-Hispanic black/ African American, and $19.6 \%$ Hispanic) (Table 1). Overall, $30.2 \%$ of respondents had been diagnosed within the past 5 years and $36.9 \%$ had been diagnosed more than 16 years ago. A total of $56.6 \%$ of respondents reported some degree of sleep difficulties, with $33.7 \%$ reporting such difficulties as moderate or severe (Fig. S1 in the supplementary material). Similarly, $70.7 \%$ of respondents reported having some form of depression based on the PHQ-9 instrument; $46.6 \%$ reported having moderateto-severe depression. Finally, $60.9 \%$ of respondents reported having anxiety.

\section{Sleep Difficulties and Health Outcomes}

Increasing severity of sleep difficulties was associated with decreased levels of health status (Fig. 1). Among respondents without sleep difficulties, mental (37.66) and physical (42.46) component summary scores were lower than the population norm of 50, with lower scores 
Table 1 Demographics and health history characteristics of the sample

\begin{tabular}{|c|c|}
\hline Characteristic & $\begin{array}{l}\text { Total } \\
\text { sample } \\
N=1017\end{array}$ \\
\hline Age, mean (SD), years & $37.4(14.5)$ \\
\hline \multicolumn{2}{|l|}{ Sex, $n(\%)$} \\
\hline Female & $749(73.6)$ \\
\hline \multicolumn{2}{|l|}{ Race/ethnicity, $n$ (\%) } \\
\hline Non-Hispanic white & $426(41.9)$ \\
\hline Non-Hispanic African American/black & $176(17.3)$ \\
\hline Hispanic & $199(19.6)$ \\
\hline Other & $216(21.2)$ \\
\hline \multicolumn{2}{|l|}{ Marital status, $n(\%)$} \\
\hline Married/living with partner & $511(50.2)$ \\
\hline Not married & $503(49.5)$ \\
\hline Missing/unknown & $3(0.3)$ \\
\hline \multicolumn{2}{|l|}{ Education level, $n$ (\%) } \\
\hline$<$ University degree & $571(56.1)$ \\
\hline$\geq$ University degree & $443(43.6)$ \\
\hline Missing/unknown & $3(0.3)$ \\
\hline \multicolumn{2}{|l|}{ Household income, $n(\%)$} \\
\hline$<\$ 25 \mathrm{k}$ & $215(21.1)$ \\
\hline$\$ 25 \mathrm{k}$ to $<\$ 50 \mathrm{k}$ & $269(26.5)$ \\
\hline$\$ 50 \mathrm{k}$ to $<\$ 75 \mathrm{k}$ & $182(17.9)$ \\
\hline$\$ 75 \mathrm{k}$ to $<\$ 100 \mathrm{k}$ & $137(13.5)$ \\
\hline$\geq \$ 100 \mathrm{k}$ & $177(17.4)$ \\
\hline Missing/unknown & $37(3.6)$ \\
\hline Currently employed, $n$ (\%) & $616(60.6)$ \\
\hline \multicolumn{2}{|l|}{ Body mass index, $n$ (\%) } \\
\hline$<18.5 \mathrm{~kg} / \mathrm{m}^{2}$ (underweight) & $36(3.5)$ \\
\hline $\begin{array}{l}18.5 \mathrm{~kg} / \mathrm{m}^{2} \text { to }<25 \mathrm{~kg} / \mathrm{m}^{2} \text { (normal } \\
\text { weight) }\end{array}$ & $357(35.1)$ \\
\hline $25 \mathrm{~kg} / \mathrm{m}^{2}$ to $<30 \mathrm{~kg} / \mathrm{m}^{2}$ (overweight) & $269(26.5)$ \\
\hline$\geq 30 \mathrm{~kg} / \mathrm{m}^{2}$ (obese) & $325(32.0)$ \\
\hline Missing/unknown & $30(2.9)$ \\
\hline
\end{tabular}

Table 1 continued

\begin{tabular}{lc}
\hline Characteristic & $\begin{array}{c}\text { Total sample } \\
N=\mathbf{1 0 1 7}\end{array}$ \\
\hline Smoking status, $n$ (\%) & $214(21.0)$ \\
Currently smoke & $223(21.9)$ \\
Former smoker & $580(57.0)$ \\
Never smoker & $683(67.2)$ \\
Currently drink, $n$ (\%) & \\
Charlson comorbidity index, $n$ (\%) & $773(76.0)$ \\
0 & $126(12.4)$ \\
1 & $58(5.7)$ \\
2 & $60(5.9)$ \\
$\geq 3$ & \\
Years since eczema diagnosis, $n(\%)$ & $307(30.2)$ \\
$<5$ years & $172(16.9)$ \\
$6-10$ years & $113(11.1)$ \\
$11-15$ years & $375(36.9)$ \\
$\geq 16$ years & $50(4.9)$ \\
\hline Missing/unknown & \\
\hline
\end{tabular}

$S D$ standard deviation

suggesting worse health. The burden increased with the severity of sleep difficulties, with the largest effects observed for mental component summary scores (29.13 vs 37.66 for severe and no sleep difficulties, respectively; $P<0.05$ ), emotional role limitations (27.17 vs 33.89 ; $P<0.05)$, social functioning (29.57 vs 36.69 ; $P<0.05$ ), physical role limitations (30.88 vs 35.32; $P<0.05)$, and mental health $(31.63$ vs 40.14; $P<0.05)$ domains.

Increasing severity of sleep difficulties was also significantly associated with poorer generic HRQoL and economic outcomes (Table S1 in the supplementary material). Respondents with severe sleep difficulties reported significantly worse health utilities ( 0.51 vs 0.67 for severe 
and no sleep difficulties, respectively; $P<0.05$ ), greater overall work impairment $(54.71 \%$ vs $44.25 \% ; P<0.05)$, greater activity impairment (65.34\% vs $48.27 \% ; P<0.05)$, more physician visits ( 17.66 vs $11.19 ; P<0.05)$, and more ER visits $(1.91$ vs $1.02 ; P<0.05)$ than respondents without sleep difficulties. No differences were observed with respect to hospitalizations.

\section{Depression and Health Outcomes}

As depression severity increased, poorer levels of health status were observed (Fig. 2). The effects were strongest for mental component summary scores ( 22.22 vs 45.31 for severe depression and none/minimal depression, respectively; $P<0.05)$, emotional role limitations (20.75 vs 41.33; $P<0.05)$, social functioning $(25.54$ vs 43.64; $P<0.05)$, mental health $(26.12$ vs 48.07 ; $P<0.05)$, and physical role limitations $(29.48$ vs 42.12; $P<0.05$ ) domains. Increasing depression severity was also associated with significantly worse health utilities $(0.49$ vs 0.75 for severe depression and none/minimal depression, respectively; $P<0.05)$, greater overall work impairment $(76.49 \%$ vs $42.42 \%$; $P<0.05)$, greater activity impairment $(70.81 \%$ vs $33.87 \%$; $P<0.05)$, more physician visits $(17.74$ vs 7.72 ; $P<0.05)$, more ER visits $(2.94$ vs 0.61 ; $P<0.05)$, and more hospitalizations (1.43 vs $1.14 ; P<0.05)$ than for respondents with minimal or no depression (Table S2 in the supplementary material).

\section{Anxiety and Health Outcomes}

Respondents with anxiety reported significantly worse health status than respondents without anxiety, with the strongest effects observed for the mental component summary score (33.52 vs 41.70; $P<0.05)$, emotional role limitations (32.40 vs $36.87 ; P<0.05)$, social functioning (36.61 vs $41.13 ; P<0.05$ ), and mental health domains (36.94 vs 45.14; $P<0.05$ ) (Fig. 3 ). Respondents with anxiety also reported significantly worse health utilities (0.68 vs 0.76 ; $P<0.05$ ) (Table S3 in the supplementary material), more overall work impairment $(57.63 \%$ vs $51.95 \% ; P<0.05)$, more activity impairment
(46.10\% vs $37.38 \% ; P<0.05$ ), and more physician visits in the past 6 months $(9.04$ vs 6.50 ; $P<0.05)$ than respondents without anxiety. No differences were observed with respect to absenteeism, presenteeism, or number of ER visits; respondents with anxiety reported fewer hospitalizations than respondents without anxiety $(0.76$ vs $1.15 ; P<0.05)$.

\section{DISCUSSION}

The aim of this study was to establish the prevalence of psychosocial comorbidities and their impact on health outcomes among adults with moderate-to-severe AD in the USA. More than half of the respondents reported sleep difficulties, and one-third reported these difficulties to be moderate or severe. These results are consistent with previously reported rates of sleep disturbance among adults with AD [19]. More than two-thirds of the respondents in this study experienced some form of depression, and nearly half met PHQ-9 criteria for moderate-tosevere depression. Approximately $40 \%$ of respondents reported having anxiety. These rates of depression and anxiety are higher than those reported in the literature, although many prior studies focused on the entire AD population (including mild disease) rather than only moderate-to-severe $\mathrm{AD}[6,20,21]$. Regardless, our results further support the high frequency of psychosocial comorbidities among patients with $\mathrm{AD}$ and indicate the importance of screening patients with moderate-to-severe $\mathrm{AD}$ for symptoms of sleep disturbance, depression, and anxiety.

Our results also suggest a significant relationship between psychosocial comorbidities and health outcomes. Increasing severity of sleep disturbances was associated with worse health status, particularly for mental healthrelated domains, increased impairment during work and daily activities, and increased HRU. These findings are consistent with prior research that identified an interaction between eczema and fatigue, sleepiness, and insomnia [22]. Similarly, a systematic review identified several relationships between sleep disturbance and daily activities and HRQoL among adults 


\begin{tabular}{|c|c|c|c|c|}
\hline & \multicolumn{2}{|c|}{$\begin{array}{l}\text { Highest mean score } \\
\text { (less severe impact) }\end{array}$} & \multicolumn{2}{|c|}{$\begin{array}{l}\text { Lowest mean score } \\
\text { (most severe impact) }\end{array}$} \\
\hline & \multicolumn{4}{|c|}{ Severity of Sleep Difficulties } \\
\hline & No Sleep Difficulties & Mild & Moderate & Severe \\
\hline MCS & 37.66 & $34.18^{*}$ & $31.24^{*}$ & $29.13^{*}$ \\
\hline PCS & 42.46 & 42.11 & $40.74^{*}$ & $39.10^{*}$ \\
\hline Bodily pain & 40.81 & $38.75^{\star}$ & $36.99^{*}$ & $34.86^{*}$ \\
\hline Vitality & 43.94 & $40.14^{*}$ & $37.93^{*}$ & $37.27^{*}$ \\
\hline General health & 43.39 & $40.94^{*}$ & $38.16^{*}$ & $37.40^{*}$ \\
\hline Physical functioning & 42.90 & 43.50 & 41.88 & $39.47^{*}$ \\
\hline Physical role limitations & 35.32 & 34.71 & $33.08^{*}$ & $30.88^{*}$ \\
\hline Emotional role limitations & 33.89 & 31.86 & $29.80^{*}$ & $27.17^{*}$ \\
\hline Mental health & 40.14 & $37.57^{*}$ & $33.92^{*}$ & $31.63^{*}$ \\
\hline Social functioning & 36.69 & $34.38^{*}$ & $32.36^{*}$ & $29.57^{*}$ \\
\hline
\end{tabular}

Fig. 1 Heatmap of the SF-36v2 component summary and domain scores by the severity of sleep difficulties. Adjusted means were calculated on the basis of the results of generalized linear models that controlled for age, sex, race/ ethnicity, education, income, employment status, body mass

with $\mathrm{AD}$ [19]. Our results extend these findings by showing that the burden experienced by patients increases concomitantly with the severity of sleep difficulties, suggesting that sleep difficulties also impact economic-related outcomes such as presenteeism, ER visits, and hospitalizations.

Anxiety and depression were also significantly associated with poorer health status and HRQoL, and with increased work impairment, activity impairment, and HRU. Prior research has highlighted the presence of anxiety and depression in moderate-to-severe $\mathrm{AD}$ [23]; however, this study modeled the specific impact of these psychosocial comorbidities. Respondents with severe depression reported 80\% more work-related impairment, twice the number of physician visits, and more than four times the number of ER visits than respondents with minimal or no depression. These rates were notably higher than the rates of respondents with depression and without $\mathrm{AD}$ using the same index, smoking status, alcohol use, Charlson comorbidity index, and the presence of other atopic conditions. ${ }^{*} P<0.05$ relative to patients with no sleep difficulties. MCS mental component summary, PCS physical component summary, SF-36v2 36-item Short Form Health Survey, version 2

data set [24]. Anxiety was also associated with poorer health status and overall HRQoL, but economic endpoints were relatively more modest, with approximately $25 \%$ more workrelated impairment and 50-90\% more HRU reported by respondents with anxiety than by those without. Our assessment of anxiety was presence versus absence in the past 12 months. Future research should explore whether the results would be different with a more granular measure of anxiety levels.

The findings of the current study suggest a large incremental effect for patients with psychosocial comorbidities. Research has suggested that such comorbidities are more frequent among those with poor disease control [25]. Therefore, improved disease management may alleviate these psychosocial comorbidities. For example, clinical trial results suggest that effective $\mathrm{AD}$ therapies can reduce levels of anxiety, depression, and sleep disturbances, presumably through the mechanism of 


\begin{tabular}{|l|c|c|c|c|c|}
\cline { 2 - 5 } \multicolumn{1}{c|}{} & \multicolumn{2}{c}{$\begin{array}{l}\text { Highest mean score } \\
\text { (less severe impact) }\end{array}$} \\
\cline { 2 - 6 } \multicolumn{1}{c|}{} & \multicolumn{3}{c|}{$\begin{array}{c}\text { Lowest mean score } \\
\text { (most severe impact) }\end{array}$} \\
\cline { 2 - 6 } & None/Minimal & Mild & Moderate & Moderately Severe & Severe \\
\hline MCS & 45.31 & $37.05^{*}$ & $32.53^{*}$ & $28.76^{*}$ & $22.22^{*}$ \\
\hline PCS & 47.49 & $45.57^{*}$ & $43.10^{*}$ & $41.59^{*}$ & $41.45^{*}$ \\
\hline Bodily pain & 45.86 & $42.08^{*}$ & $39.56^{*}$ & $37.31^{*}$ & $35.17^{*}$ \\
\hline Vitality & 50.62 & $44.26^{*}$ & $41.14^{*}$ & $40.24^{*}$ & $35.83^{*}$ \\
\hline General health & 49.04 & $44.35^{*}$ & $41.41^{*}$ & $40.36^{*}$ & $37.43^{*}$ \\
\hline Physical functioning & 49.20 & $46.35^{*}$ & $43.01^{*}$ & $40.20^{*}$ & $40.18^{*}$ \\
\hline Physical role limitations & 42.12 & $38.74^{*}$ & $34.75^{*}$ & $31.77^{*}$ & $29.48^{*}$ \\
\hline Emotional role limitations & 41.33 & $35.37^{*}$ & $29.87^{*}$ & $24.73^{*}$ & $20.75^{*}$ \\
\hline Mental health & 48.07 & $39.80^{*}$ & $35.75^{*}$ & $32.53^{*}$ & $26.12^{*}$ \\
\hline Social functioning & 43.64 & $37.43^{*}$ & $33.78^{*}$ & $30.00^{*}$ & $25.54^{*}$ \\
\hline
\end{tabular}

Fig. 2 Heatmap of the association between depression severity and health status. Adjusted means were calculated on the basis of the results of generalized linear models that controlled for age, sex, race/ethnicity, education, income, employment status, body mass index, smoking status,

improving the signs and symptoms of the disease [26]. These findings reinforce the potential secondary benefits of adequate $\mathrm{AD}$ management because the improvement in the psychosocial health of patients may benefit not only the patient but also caregivers, employers, and the healthcare system.

The data from the NHWS is entirely respondent-reported, without any verification (clinician or otherwise) of responses. Recall error or other response biases may have been introduced. However, in previous research, self-report of AD has been reliable [27]. The definition of severity was based on DLQI bands. Using alternative definitions, such as treatment history, Eczema Area and Severity Index, or percentage of body surface area affected, may have generated different findings. Because of the cross-sectional nature of the data, any relationships uncovered in the study can only be viewed as associations because the presence and direction of causality cannot be known. The alcohol use, Charlson comorbidity index, and the presence of other atopic conditions. ${ }^{*} P<0.05$ relative to respondents with none/minimal depression. MCS mental component summary, PCS physical component summary, PHQ-9 Patient Health Questionnaire-9

study relied on multivariable models to rule out alternative explanations. It is possible that variables that could partially explain the relationships observed here were not available for inclusion in these models. Finally, the current sample was recruited from the NHWS. Although the demographic composition mimics the age, sex, and race/ethnicity distribution of that in the USA, it is possible that the specific $A D$ sample in the current study may not be truly representative of the respective $\mathrm{AD}$ population.

\section{CONCLUSION}

Psychosocial comorbidities (i.e., sleep difficulties, depression, and anxiety) were significantly associated with decrements in health status and HRQoL and significantly associated with workrelated impairment and HRU in respondents with moderate-to-severe AD. Reducing the burden of these psychosocial comorbidities in $\mathrm{AD}$ 


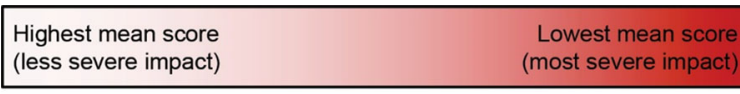

\begin{tabular}{|l|c|c|}
\cline { 2 - 3 } \multicolumn{1}{c|}{} & No Anxiety & Anxiety \\
\hline MCS & 41.70 & $33.52^{*}$ \\
\hline PCS & 46.65 & 47.67 \\
\hline Bodily pain & 45.41 & $43.59^{*}$ \\
\hline Vitality & 48.67 & $43.22^{*}$ \\
\hline General health & 48.01 & $44.45^{*}$ \\
\hline Physical functioning & 47.26 & 47.47 \\
\hline Physical role limitations & 39.00 & 38.60 \\
\hline Emotional role limitations & 36.87 & $32.40^{*}$ \\
\hline Mental health & 45.14 & $36.94^{*}$ \\
\hline Social functioning & 41.13 & $36.61^{*}$ \\
\hline
\end{tabular}

Fig. 3 Heatmap of the association between the presence of anxiety and health status. Adjusted means were calculated on the basis of the results of generalized linear models that controlled for age, sex, race/ethnicity, education, income, employment status, body mass index, smoking status, alcohol use, Charlson comorbidity index, and the presence of other atopic conditions. ${ }^{*} P<0.05$ relative to respondents with no anxiety. MCS mental component summary, PCS physical component summary

could have significant benefit to patients, caregivers, employers, and the healthcare system.

\section{ACKNOWLEDGEMENTS}

The authors thank Mamta B. Jhaveri, MD, for her contributions to the development of this manuscript.

Funding. This study was funded by Pfizer Inc., and the Journal's rapid service and open access fees were provided by Pfizer Inc.

Authorship. All named authors meet the International Committee of Medical Journal Editors (ICMJE) criteria for authorship for this article, take responsibility for the integrity of the work as a whole, and have given their approval for this version to be published.
Author contributions. All authors contributed to the conception of the manuscript, performed data analysis, critically revised the work, and edited the final draft.

Medical Writing and/or Editorial Assistance. Medical writing support under the guidance of the authors was provided by Juan Sanchez-Cortes, PhD, at ApotheCom, San Francisco, CA, USA, and was funded by Pfizer Inc., New York, NY, USA, in accordance with Good Publication Practice (GPP3) guidelines (Ann Intern Med. 2015;163:461-464).

Prior Presentation. A portion of these data has been previously presented at the American Academy of Dermatology 2020 Annual Meeting; March 20-24, 2020; Denver, Colorado. Kwatra SG, Huang AH, Jhaveri MB, Gruben D, Fung S, DiBonaventura M. Prevalence and Impact of Psychosocial Comorbidities on Health Status Among Patients With Moderateto-Severe Atopic Dermatitis (AD) in the United States: Analysis of the 2017 US National Health and Wellness Survey (NHWS).

Disclosures. Shawn G. Kwatra has received grants, personal fees, and/or nonfinancial support from Pfizer Inc., Kiniksa Pharmaceuticals, Menlo Therapeutics, and Regeneron Pharmaceuticals. David Gruben, Selwyn Fung, and Marco DiBonaventura are employees and stockholders of Pfizer Inc.

Compliance with Ethics Guidelines. The institutional review board at each study site approved the study protocol, and written informed consent was provided by parents/legal guardians. The study was conducted in accordance with the protocol, local legal and regulatory requirements, and the general principles set forth in the International Ethical Guidelines for Biomedical Research Involving Human Subjects, International Conference on Harmonisation Guideline for Good Clinical Practice, and the Declaration of Helsinki. The protocol and study materials associated with the original fielding of the 2017 NHWS were reviewed by the Pearl Institutional Review Board (Indianapolis, IN) and granted exemption 
status. Written informed consent was provided by participants of all studies.

Data Availability. Upon request, and subject to certain criteria, conditions and exceptions (see https://www.pfizer.com/science/ clinical-trials/trial-data-and-results for more information), Pfizer will provide access to individual de-identified participant data from Pfizersponsored global interventional clinical studies conducted for medicines, vaccines, and medical devices (1) for indications that have been approved in the USA and/or EU or (2) in programs that have been terminated (i.e., development for all indications has been discontinued). Pfizer will also consider requests for the protocol, data dictionary, and statistical analysis plan. Data may be requested from Pfizer trials 24 months after study completion. The deidentified participant data will be made available to researchers whose proposals meet the research criteria and other conditions, and for which an exception does not apply, via a secure portal. To gain access, data requestors must enter into a data access agreement with Pfizer.

Open Access. This article is licensed under a Creative Commons Attribution-NonCommercial 4.0 International License, which permits any non-commercial use, sharing, adaptation, distribution and reproduction in any medium or format, as long as you give appropriate credit to the original author(s) and the source, provide a link to the Creative Commons licence, and indicate if changes were made. The images or other third party material in this article are included in the article's Creative Commons licence, unless indicated otherwise in a credit line to the material. If material is not included in the article's Creative Commons licence and your intended use is not permitted by statutory regulation or exceeds the permitted use, you will need to obtain permission directly from the copyright holder. To view a copy of this licence, visit http://creativecommons.org/licenses/by$\mathrm{nc} / 4.0 /$.

\section{REFERENCES}

1. Boguniewicz M, Fonacier L, Guttman-Yassky E, Ong PY, Silverberg J, Farrar JR. Atopic dermatitis yardstick: practical recommendations for an evolving therapeutic landscape. Ann Allergy Asthma Immunol. 2018;120(1):10-22.e2.

2. Odhiambo JA, Williams HC, Clayton TO, Robertson $\mathrm{CF}$, Asher MI. Global variations in prevalence of eczema symptoms in children from ISAAC phase three. J Allergy Clin Immunol. 2009;124(6):1251-8. e23.

3. Silverberg JI, Hanifin JM. Adult eczema prevalence and associations with asthma and other health and demographic factors: a US population-based study. J Allergy Clin Immunol. 2013;132(5):1132-8.

4. Eckert L, Gupta S, Amand C, Gadkari A, Mahajan P, Gelfand JM. Impact of atopic dermatitis on healthrelated quality of life and productivity in adults in the United States: an analysis using the National Health and Wellness Survey. J Am Acad Dermatol. 2017;77(2):274-9.e3.

5. Yu SH, Attarian H, Zee P, Silverberg JI. Burden of sleep and fatigue in US adults with atopic dermatitis. Dermatitis. 2016;27(2):50-8.

6. $\mathrm{Yu} \mathrm{SH,} \mathrm{Silverberg} \mathrm{JI.} \mathrm{Association} \mathrm{between} \mathrm{atopic}$ dermatitis and depression in US adults. J Investig Dermatol. 2015;135(12):3183-6.

7. Ronnstad ATM, Halling-Overgaard AS, Hamann CR, Skov L, Egeberg A, Thyssen JP. Association of atopic dermatitis with depression, anxiety, and suicidal ideation in children and adults: a systematic review and meta-analysis. J Am Acad Dermatol. 2018;79(3):448-56.e30.

8. Drucker AM, Qureshi AA, Amand C, et al. Health care resource utilization and costs among adults with atopic dermatitis in the United States: a claims-based analysis. J Allergy Clin Immunol Pract. 2018;6(4):1342-8.

9. Eichenfield LF, DiBonaventura M, Xenakis J, et al. Costs and treatment patterns among patients with atopic dermatitis using advanced therapies in the United States: analysis of a retrospective claims database. Dermatol Ther (Heidelb). 2020;10(4): 791-806.

10. Shrestha S, Miao R, Wang L, Chao J, Yuce H, Wei W. Burden of atopic dermatitis in the United States: analysis of healthcare claims data in the commercial, Medicare, and Medi-Cal databases. Adv Ther. 2017;34(8):1989-2006. 
11. Nørreslet LB, Ebbehoj NE, Ellekilde Bonde JP, Thomsen SF, Agner T. The impact of atopic dermatitis on work life-a systematic review. J Eur Acad Dermatol Venereol. 2018;32(1):23-38.

12. United States Census Bureau. Current population survey. https://www.census.gov/programs-surveys/ cps/data-detail.html. Accessed 25 Mar 2020.

13. Finlay AY, Khan GK. Dermatology Life Quality Index (DLQI) - a simple practical measure for routine clinical use. Clin Exp Dermatol. 1994;19(3): 210-6.

14. Quan H, Li B, Couris CM, Fushimi K, et al. Updating and validating the Charlson comorbidity index and score for risk adjustment in hospital discharge abstracts using data from 6 countries. Am J Epidemiol. 2011;173(6):676-82.

15. Kroenke K, Spitzer RL, Williams JB. The PHQ-9: validity of a brief depression severity measure. J Gen Intern Med. 2001;16(9):606-13.

16. Ware J, Kosinski M, Bjorner J, Turner-Bowker D, Gandek B, Maruish M. User's manual for the SF36v2 ${ }^{\circledR}$ Health Survey. Lincoln: QualityMetric; 2007.

17. Herdman M, Gudex C, Lloyd A, et al. Development and preliminary testing of the new five-level version of EQ-5D (EQ-5D-5L). Qual Life Res. 2011;20(10):1727-36.

18. Reilly MC, Zbrozek AS, Dukes EM. The validity and reproducibility of a work productivity and activity impairment instrument. Pharmacoeconomics. 1993;4(5):353-65.

19. Jeon C, Yan D, Nakamura M, et al. Frequency and management of sleep disturbance in adults with atopic dermatitis: a systematic review. Dermatol Ther (Heidelb). 2017;7(3):349-64.
20. Cheng BT, Silverberg JI. Depression and psychological distress in US adults with atopic dermatitis. Ann Allergy Asthma Immunol. 2019;123(2): 179-85.

21. Silverberg JI, Gelfand JM, Margolis DJ, et al. Symptoms and diagnosis of anxiety and depression in atopic dermatitis in US adults. Br J Dermatol. 2019;181(3):554-65.

22. Silverberg JI, Garg NK, Paller AS, Fishbein AB, Zee PC. Sleep disturbances in adults with eczema are associated with impaired overall health: a US population-based study. J Investig Dermatol. 2015;135(1):56-66.

23. Simpson EL, Guttman-Yassky E, Margolis DJ, et al. Association of inadequately controlled disease and disease severity with patient-reported disease burden in adults with atopic dermatitis. JAMA Dermatol. 2018;154(8):903-12.

24. Gupta S, Goren A, Kim E, Gabriel S, Dupclay L. Direct and indirect costs of sleep disturbances and residual symptoms in patients treated for depression: analysis of a nationwide cross sectional survey. J Occup Environ Med. 2012;54(7):881-8.

25. Wei W, Anderson P, Gadkari A, et al. Extent and consequences of inadequate disease control among adults with a history of moderate to severe atopic dermatitis. J Dermatol. 2018;45(2):150-7.

26. Simpson EL, Bieber T, Guttman-Yassky E, et al. Two phase 3 trials of dupilumab versus placebo in atopic dermatitis. N Engl J Med. 2016;375(24):2335-48.

27. Silverberg JI, Patel N, Immaneni S, et al. Assessment of atopic dermatitis using self-report and caregiver report: a multicentre validation study. Br J Dermatol. $2015 ; 173(6): 1400-4$. 